\title{
Magnetic Field Frequency Optimisation for MFL Imaging using QWHE Sensors
}

Link to publication record in Manchester Research Explorer

\section{Citation for published version (APA):}

Watson, J. M., Liang, C-W., Sexton, J., \& Missous, M. (2019). Magnetic Field Frequency Optimisation for MFL Imaging using QWHE Sensors. Paper presented at 58th Annual British Conference on Non-Destructive Testing, Telford, United Kingdom.

\section{Citing this paper}

Please note that where the full-text provided on Manchester Research Explorer is the Author Accepted Manuscript or Proof version this may differ from the final Published version. If citing, it is advised that you check and use the publisher's definitive version.

\section{General rights}

Copyright and moral rights for the publications made accessible in the Research Explorer are retained by the authors and/or other copyright owners and it is a condition of accessing publications that users recognise and abide by the legal requirements associated with these rights.

\section{Takedown policy}

If you believe that this document breaches copyright please refer to the University of Manchester's Takedown Procedures [http://man.ac.uk/04Y6Bo] or contact uml.scholarlycommunications@manchester.ac.uk providing relevant details, so we can investigate your claim.

\section{OPEN ACCESS}




\section{Magnetic Field Frequency Optimisation for MFL Imaging using QWHE Sensors}

\begin{tabular}{|c|c|}
\hline Journal: & 58th Annual British Conference on Non-Destructive Testing \\
\hline Manuscript ID & NDT-0028-2019.R1 \\
\hline Topic: & Electromagnetics \\
\hline $\begin{array}{r}\text { Date Submitted by the } \\
\text { Author: }\end{array}$ & $\mathrm{n} / \mathrm{a}$ \\
\hline Complete List of Authors: & $\begin{array}{l}\text { Watson, James; University of Manchester, School of Electrical and } \\
\text { Electronic Engineering }\end{array}$ \\
\hline Keywords: & $\begin{array}{l}\text { Electromagnetics, Magnetic flux leakage, Magnetic particles, Eddy } \\
\text { currents, Image formation, Frequencies }\end{array}$ \\
\hline
\end{tabular}

\section{SCHOLARONE \\ Manuscripts}




\title{
Magnetic Field Frequency Optimisation for MFL Imaging using QWHE Sensors
}

\author{
J. M. Watson, C.W. Liang, J. Sexton and M. Missous \\ University of Manchester, Department of Electrical and Electronic Engineering \\ Manchester, M13 9PL, United Kingdom \\ james.watson-6@postgrad.manchester.ac.uk
}

\begin{abstract}
Magnetic particle and other magnetic flux leakage-based methods for the detection and evaluation of surface-breaking flaws in ferromagnetic materials typically use highstrength $(\sim 0.5 \mathrm{~T} \mathrm{rms})$, low frequency $(\leq 50 \mathrm{~Hz})$ magnetic fields. The rationale behind this is the ready availability of strong permanent magnets and mains power to create high strength electromagnets. This high field strength is needed to saturate the sample and compensate for the insensitivity of magnetic particles, silicon Hall sensors, coils and other magnetic transducers.
\end{abstract}

As such, the frequency of applied magnetic field is typically limited to $\leq 50 \mathrm{~Hz}$ and does not take into account the frequency response of the material under test (some MFL applications use this low frequency to detect sub-surface or flaws on the back wall).

In this study, a probe consisting of a Quantum Well Hall Effect (QWHE) sensor, illuminating electromagnet and sensor circuitry was controlled using an automated XYZ scanner with an $\mathrm{x} y$ measurement step size (i.e. magnetic image pixel size) of 100 microns. This probe was used to apply a magnetic field of various frequencies (DC to 1 $\mathrm{kHz})$ and field strengths ( $5 \mathrm{mT}$ to $100 \mathrm{mT}$ ) to ascertain a frequency and field range best suited to detecting 10 and $11 \mathrm{~mm}$ length longitudinal surface-breaking toe cracks in ground mild steel welds. A lift-off distance of $<1 \mathrm{~mm}$ was controlled using a proximity laser and $\mathrm{z}$ direction motor module to autonomously control the probe lift-off and conform to sample geometry.

This study found that an applied magnetic field of frequency $800 \mathrm{~Hz}$ and strength 10 mT rms was optimal, based on the ratio of MFL responses from the two flaws and the weld. The power dissipation of the coil was taken into account in this determination, where other frequency-field combinations had comparable or higher detection but were discounted as they had substantially higher power consumption. 


\section{Introduction}

Complimentary electromagnetic Non Destructive Testing and Evaluation (NDT\&E) techniques are often the preferred choice for surface-breaking flaw detection and evaluation in mild steels due to the versatility and large inspection area of Magnetic Particle Inspection (MPI) ${ }^{(1)}$, as well as the depth measurement and higher sensitivity (due to the electromagnetic skin effect) of conventional Eddy Current Testing (ECT) ${ }^{(2)}$ and other eddy current-based technologies and methods ${ }^{(3)}$.

An examination of the typical frequencies of applied magnetic field, depicted in Figure 1 below, shows a distinct gap between the Magnetic Flux Leakage (MFL) and eddy current-based techniques ${ }^{(3)}$.

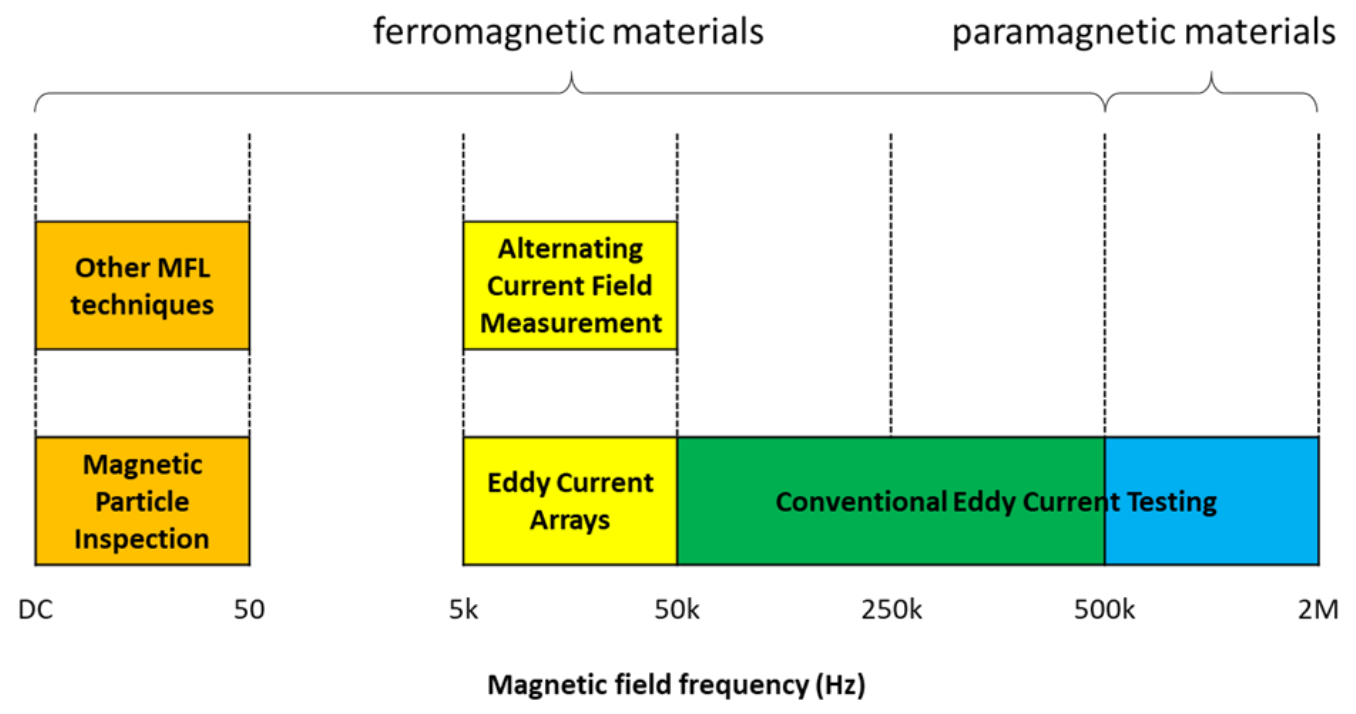

Figure 1. Typical range of applied magnetic field frequencies used for different electromagnetic NDT\&E methods.

The main reasons for this distinct gap include:

- Higher frequencies generate more eddy current based on Faraday's Law.

- Coil sensors are more sensitive at higher frequencies.

- Some MFL-based methods use a DC field to achieve full sample penetration (no electromagnetic skin effect).

- Coils, generic silicon Hall sensors and detection media are insensitive to small flaws, so large applied field strengths are required (conveniently generated using $50 \mathrm{~Hz}$ mains).

Consequently, for surface-breaking flaw MFL-based methods, such as MPI and MFL imaging, an applied magnetic field frequency of $50 \mathrm{~Hz}$ is used out of convenience and necessity for the illuminating coil power consumption requirements because of detection insensitivity (transducer or media). 
As such, typical surface-breaking flaw MFL-based methods are not truly optimised to the magnetic response of the sample material.

This study involved using Quantum Well Hall Effect (QWHE) sensors developed at The University of Manchester ${ }^{(4)}$ to ascertain if there was any optimum applied magnetic field frequency or field strength range for a particular type and size of flaws. The QWHE sensors were used because of their proven ability to detect the MFL response from real NDE flaws ${ }^{(5)}$. A previous comparative study ${ }^{(6)}{ }^{(7)}$ suggests they have a detection performance comparable to conventional ECT. This sensor system technology has high quality imaging capabilities ${ }^{(8)}$ with better flaw characterisation than eddy current-based images and has additional weld information ${ }^{(9)}$.

Key attributes of the QWHE sensors include:

- Ability to be made into bespoke arrays with specific size $(2 \mu \mathrm{m}$ to $70 \mu \mathrm{m})$ and sensor pitch $(<20 \mu \mathrm{m})$.

- Sensitive to only one component of the sample magnetic field response, i.e. $B_{z}$ in Equation 1.

- High sensitivity which is limited by biasing and detection circuit electronics (currently $20 \mathrm{nT}$ detection limit in both $\mathrm{AC}$ and DC using superheterodyne techniques).

- Large dynamic range of $20 \mathrm{nT}$ to $\sim 2 \mathrm{~T}(160 \mathrm{~dB})$ for versatility with no hysteresis offset unlike Giant Magnetoresistors (GMRs) and other anisotropic magnetoresistance-based transducers.

- Linear across large dynamic range and wide bandwidth from DC to $\mathrm{MHz}$ range.

It is the unique combination of sensitivity and linearity over a large dynamic range that make QWHE sensors versatile for different NDT\&E applications, in particular, optimised low-power high-quality (flaw characterisation) MFL imaging technology.

These novel sensor characteristics are the result of the previous 20 year development and refinement of the sensor AlGaAs-InGaAs semiconductor heterostructure where a quantum well confines electrons within a $120 \AA$ thin layer as a 2-Dimensional Electron Gas (2DEG). With additional electron supply layers and a refined doping ratio in the 2DEG layer to increase electron mobility, ultimately enabling a Hall sensor with high sensitivity and linearity over a large dynamic range with a lower resistance (thermal noise).

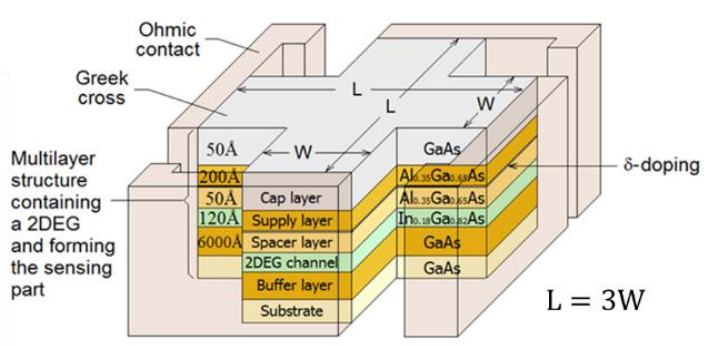

(a)

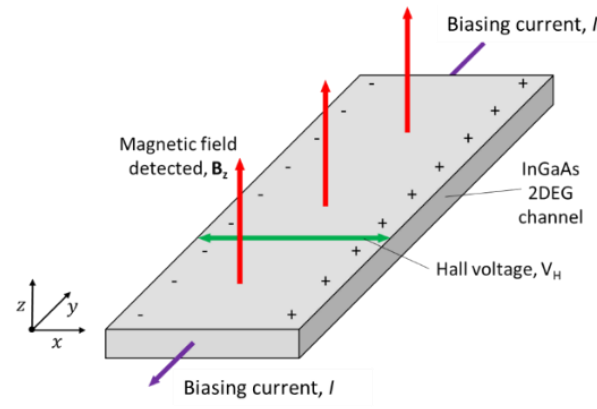

(b)

Figure 2. (a)Typical structure of a QWHE sensor. (b) Diagram of the Hall Effect. 
It must be noted that QWHE sensors operate using the "conventional" Hall effect, as described below in Equation (1); they do not use the Quantum Hall Effect or require active cooling like other Quantum-enabled transducers (i.e. SQUIDs).

$$
V_{H}=\frac{1}{t \cdot n \cdot e} \cdot I \cdot B_{z}
$$

Where $V_{H}$ is the Hall voltage, $t$ is the thickness of the 2DEG channel, $n$ is the electron concentration, $e$ is the electron charge, $I$ is the biasing current and $B_{z}$ is the component of magnetic field detected.

Equation 1 above emphasises that the thickness $t$ of the 2DEG channel and electron concentration $n$ determine the underlying sensitivity of the device. It also shows that the output Hall voltage is the vector product of the biasing current $I$ and detected magnetic field component $B_{z}$, meaning that the Hall voltage signal $V_{H}$ is a mixed combination of the biasing current of magnetic field which typically have different frequencies.

Consequently, the QWHE sensor can be operated using superheterodyne mixing to translate high frequency $(>30 \mathrm{kHz})$ magnetic field signals to lower frequencies $(<1$ $\mathrm{kHz}$ ) enabling much lower $1 / \mathrm{f}$ and thermal noise components. 


\section{MFL Imaging using QWHE Sensors}

This section provides information on the sample used in this study, as well as an overview of the QWHE sensor scanner used, the data acquisition steps of the system and the data collection procedures.

\subsection{Sample Under Test}

A ground mild steel plate weld sample manufactured by Sonaspection, with two surfacebreaking longitudinal toe cracks of lengths $10 \mathrm{~mm}$ and $11 \mathrm{~mm}$ was used in this study. Figure 3 below shows the official drawing given from Sonaspection:
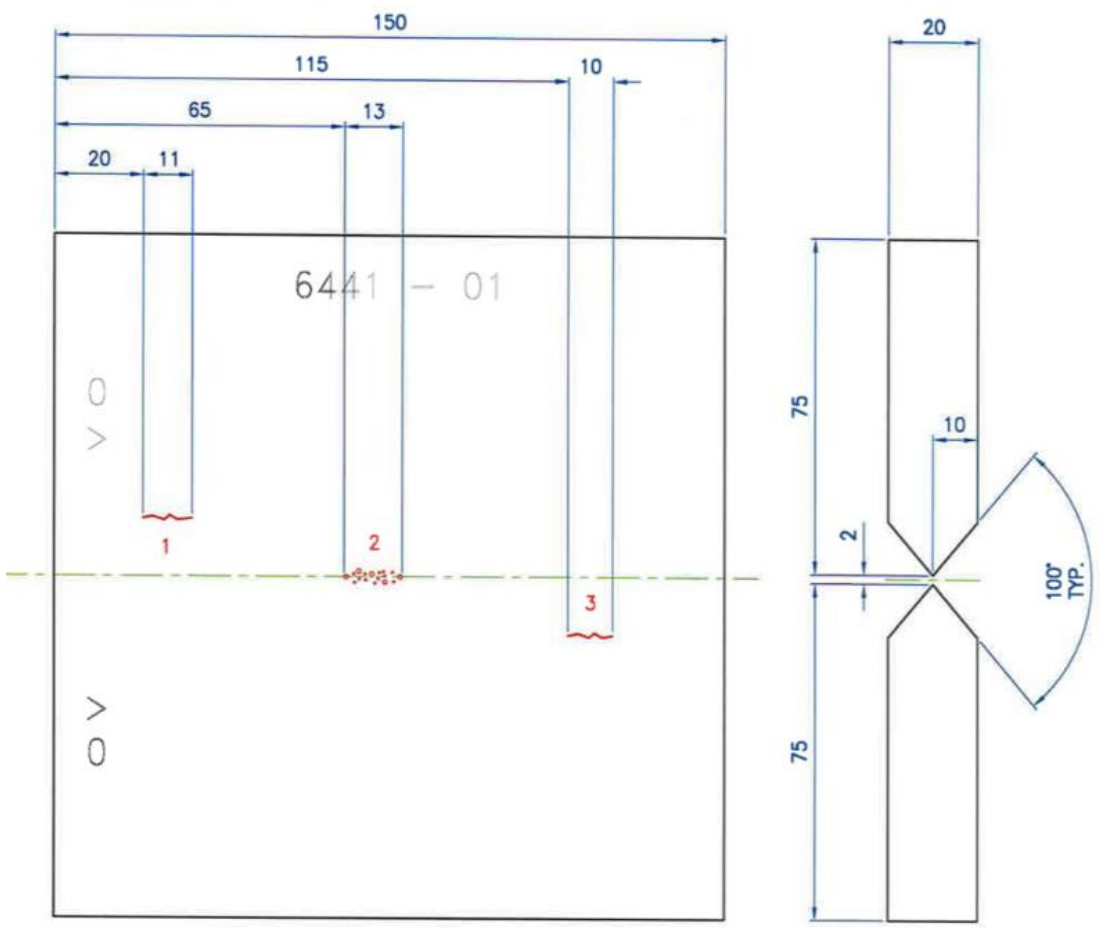

Figure 3. Sample drawing.

As Figure 3 shows, the sample measured $150 \mathrm{~mm}$ x $150 \mathrm{~mm}$ with a thickness of $20 \mathrm{~mm}$. It contained a full penetration Tungsten Inert Gas (TIG) weld whose cap and root were ground flush. The parent metal was generic mild steel, with electrical and magnetic properties similar to AISI 1010. MPI was used to validate the sizes of the flaws, however a previous study found that QWHE magnetic imaging, conventional ECT and Alternating Current Field Measurement (ACFM) values differed slightly (all within a $\pm 2 \mathrm{~mm}$ range) ${ }^{(9)}$.

The porosity (labelled as "Flaw 2" on Figure 3) on the sample was located on the root side of the sample. For this study, only the two toe cracks (i.e. "Flaw 1" and "Flaw 3" on Figure 3) on the cap side were investigated. 


\subsection{QWHE Imaging XYZ Scanner}

The developed scanner ${ }^{(6)}$ (7) autonomously controlled the fine movement of a probe consisting of a $10 \mu \mathrm{m}$ size QWHE sensor and its biasing/detection circuitry, along with an illuminating electromagnet. The electromagnet applied a magnetic field of precisely known frequency and field strength to the sample, with the QWHE sensor and data acquisition system mapping the MFL response for each $\mathrm{x}$ and $\mathrm{y}$ position on the sample surface.

A proximity laser on the probe was used to take an initial topographical scan of the region to be magnetically imaged. An additional $\mathrm{z}$ direction motor module was used to autonomously control the probe lift-off, using the laser map to compensate for changes in lift-off due to sample curvature. This was done to avoid damage to the probe head and sample, as well as provide better quality magnetic images.

\subsection{NDE Magnetic Imaging and Data Processing Procedures}

In this study, the sample was scanned using applied magnetic field frequencies and field strengths across the entire operational range of the QWHE magnetic imaging system (limited by the illuminating coil parameters). This range is shown in Table 1 below:

Table 1. Applied magnetic field frequency and field ranges used in study.

\begin{tabular}{|c|c|}
\hline $\begin{array}{c}\text { Applied magnetic } \\
\text { field frequency, } f \\
(\mathrm{~Hz})\end{array}$ & $\begin{array}{c}\text { Applied magnetic } \\
\text { field strength, } B \\
\text { (mT rms) }\end{array}$ \\
\hline 1000 & 5 \\
\hline 900 & 5 \\
\hline 800 & 10 \\
\hline 800 & 5 \\
\hline 700 & 10 \\
\hline 700 & 5 \\
\hline 600 & 10 \\
\hline 600 & 5 \\
\hline 500 & 20 \\
\hline 500 & 10 \\
\hline 500 & 5 \\
\hline 400 & 30 \\
\hline 400 & 20 \\
\hline 400 & 10 \\
\hline 400 & 5 \\
\hline 300 & 40 \\
\hline 300 & 30 \\
\hline 300 & 20 \\
\hline 300 & 10 \\
\hline 300 & 5 \\
\hline & \\
\hline & \\
\hline
\end{tabular}

\begin{tabular}{|c|c|}
\hline $\begin{array}{c}\text { Applied magnetic } \\
\text { field frequency, } f \\
(\mathrm{~Hz})\end{array}$ & $\begin{array}{c}\text { Applied magnetic } \\
\text { field strength, } B \\
\text { (mT rms) }\end{array}$ \\
\hline 200 & 50 \\
\hline 200 & 40 \\
\hline 200 & 30 \\
\hline 200 & 20 \\
\hline 200 & 10 \\
\hline 200 & 5 \\
\hline 100 & 60 \\
\hline 100 & 50 \\
\hline 100 & 40 \\
\hline 100 & 30 \\
\hline 100 & 20 \\
\hline 100 & 10 \\
\hline 100 & 5 \\
\hline DC & 10 \\
\hline DC & 20 \\
\hline DC & 30 \\
\hline DC & 40 \\
\hline DC & 60 \\
\hline DC & 80 \\
\hline DC & 100 \\
\hline & \\
\hline
\end{tabular}


The sample was firmly secured to the scanner bed to ensure no movement during the scans (particularly at DC where strong field strengths can pick up the sample). A fine measurement step of $100 \mu \mathrm{m}$ for $\mathrm{x}$ and y was used (giving a pixel size of $100 \mu \mathrm{m}$ x 100 $\mu \mathrm{m})$, with a $0.75 \mathrm{~mm}$ lift-off to ensure adequate clearance throughout the scanning.

As shown in Figure 4 below, a region of $70 \mathrm{~mm}$ (approximately central across the weld) x $140 \mathrm{~mm}$ (along the weld) was scanned:

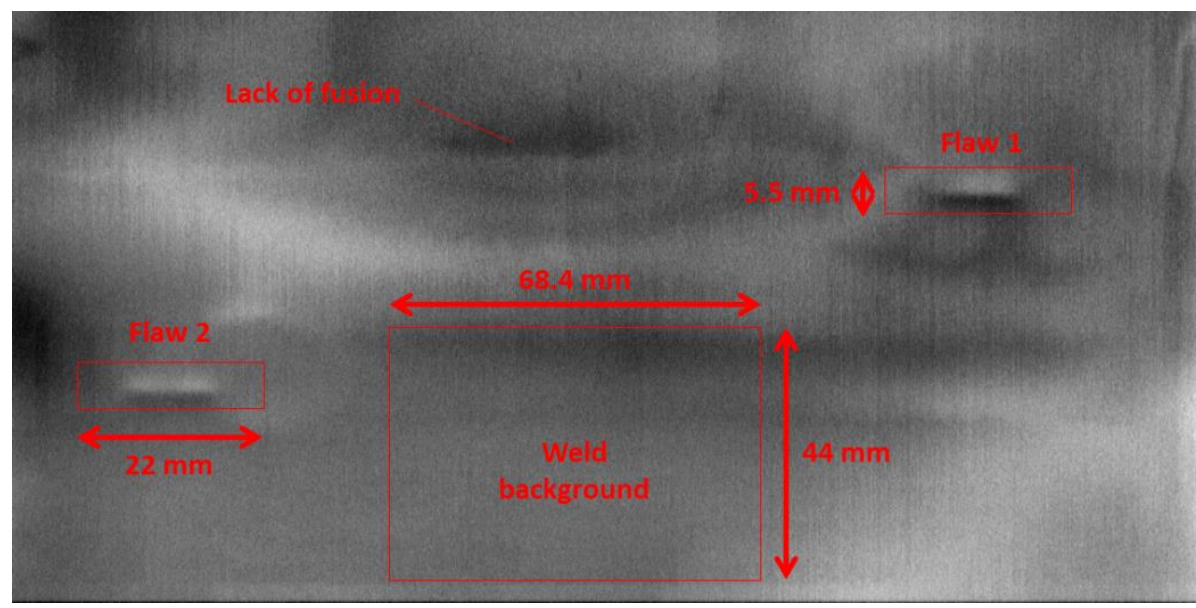

Figure 4. Example raw magnetic image showing regions of interest used for analysis.

Each raw unprocessed magnetic image, for the different magnetic field frequency and field strength combinations, was analysed to determine whether an optimal range that had higher detectability was present. This was achieved by calculating the rms pixel value $\alpha$ for each of the three regions of interest shown in Figure 4 above: Flaw 1, Flaw 2 and the background weld (i.e. $\alpha_{1} \alpha_{2} \alpha_{0}$ respectively). This calculation was extracted from Equation 2 below:

$$
\alpha_{i}=\sqrt{\frac{1}{n} \iint V_{x y}^{2} d x d y}
$$

Where $\alpha_{i}$ is the rms value for that region of interest, $n$ is the number of pixels contained within that region of interest, $\boldsymbol{V}_{\boldsymbol{x} \boldsymbol{y}}^{\boldsymbol{2}}$ is the pixel value for that (x,y) position, $\boldsymbol{d} \boldsymbol{x}$ is the $\mathrm{x}$ measurement step and $\boldsymbol{d} \boldsymbol{y}$ is the y measurement step.

From these $\alpha$ values, the MFL signal to background weld signal ratio $\beta$, was calculated in $\mathrm{dB}$ for Flaw 1 and Flaw 2 as shown in Equation 3 below. These values can be considered as a form of signal to noise ratio, but are only expressed in terms of the MFL responses from the flaw and weld and are only able to compare the images from this data set (not to be seen as the "true" signal to noise ratio of QWHE sensor technology).

$$
\beta_{i}=20 \log \left(\frac{\alpha_{i}}{\alpha_{0}}\right)
$$

Where $\beta_{i}$ is the MFL signal to background weld signal ratio. 
In addition, the mean $\beta$ value, $\bar{\beta}$, for each applied magnetic field frequency and field strength combination was calculated in $\mathrm{dB}$ as show in Equation 4 below:

$$
\bar{\beta}=20 \log \left(\frac{\alpha_{1}+\alpha_{2}}{2 \alpha_{0}}\right)
$$

The $\beta_{1}, \beta_{2}$ and $\bar{\beta}$ values for each applied magnetic field frequency and field strength combination were then plotted against applied magnetic field frequency and field strength to ascertain any optimum range. These plots are shown and discussed in the results section.

Figure 4 shows that the full width of the weld in the sample was not used in the calculations because of a suspected lack of fusion. Although the sample was validated for general magnetic testing, the previous comparative study found that ECT and ACFM also detected this feature. As such, the presence of this within the weld background signal $\alpha_{0}$ would make it not representative of a "clean" weld and would have greatly influenced the outcomes and therefore it was excluded from the calculations.

All of the scans were performed consecutively to minimise any environmental changes (different lift-off, condition of the system, magnetisation of the sample from a different measurement, etc.) that could have affected the results. In addition, the DC scans were performed at the end in ascending order to prevent a previous higher strength scan affecting the measurement via residual magnetisation of the coil core or sample. 


\section{Results and Discussion}

This study produced 40 QWHE magnetic images from the different applied magnetic field frequency and field strength combinations across the operational range of the XYZ scanner. A selection of these images are given below in Figure 5.

It must be emphasised that these are raw unprocessed images. Flaw detection, sizing and characterisation performance are dramatically increased when basic filtering and hill shading processing techniques are used (these will be reported in future communications).

(a) DC at $80 \mathrm{mT}$

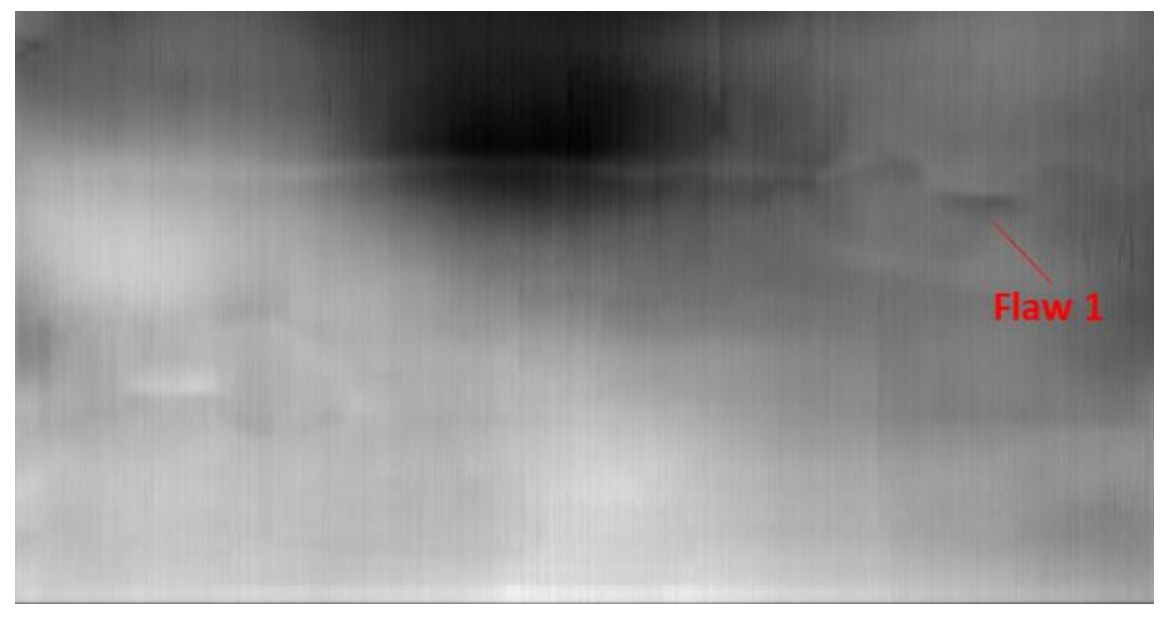

(b) $200 \mathrm{~Hz}$ at $50 \mathrm{mT}$

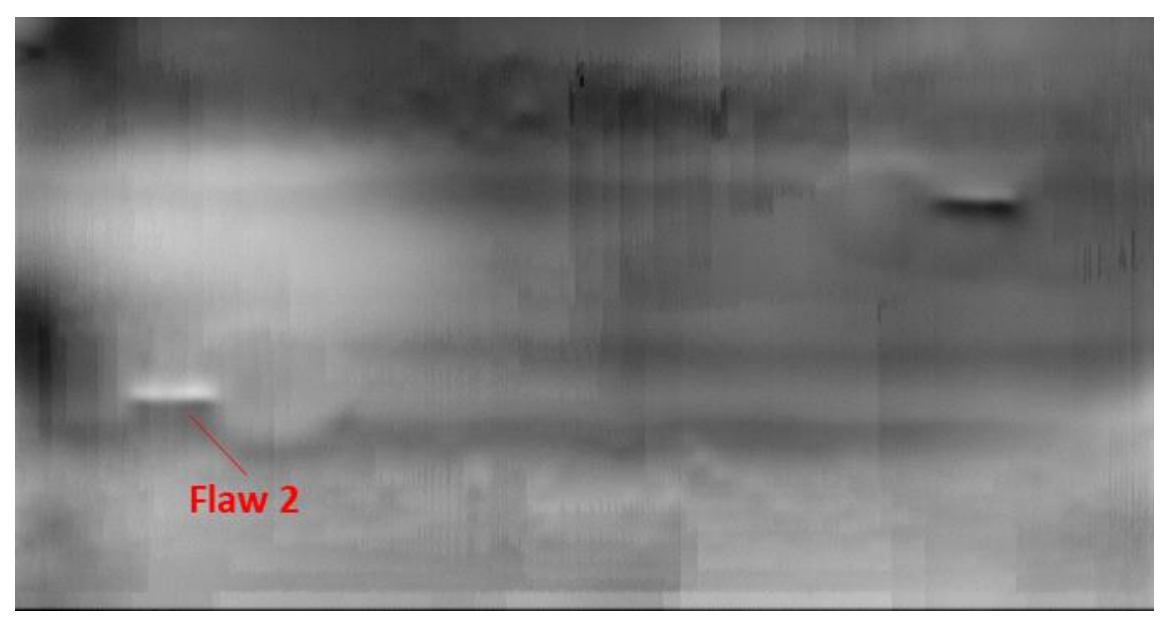


(d) $600 \mathrm{~Hz}$ at $10 \mathrm{mT}$

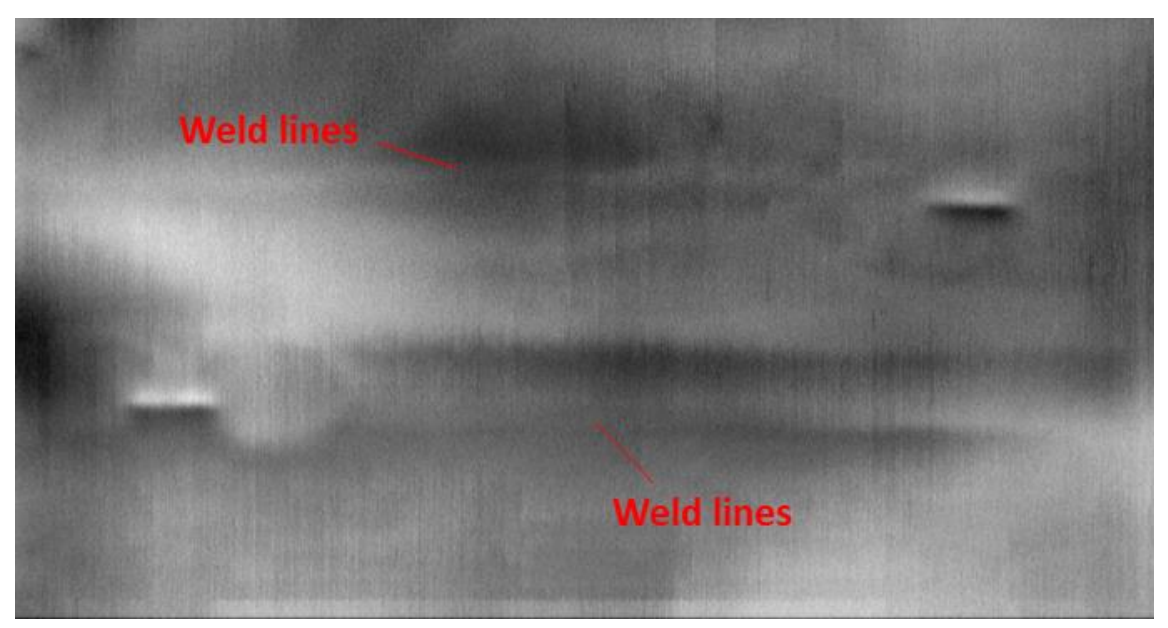

(f) $1000 \mathrm{~Hz}$ at $5 \mathrm{mT}$

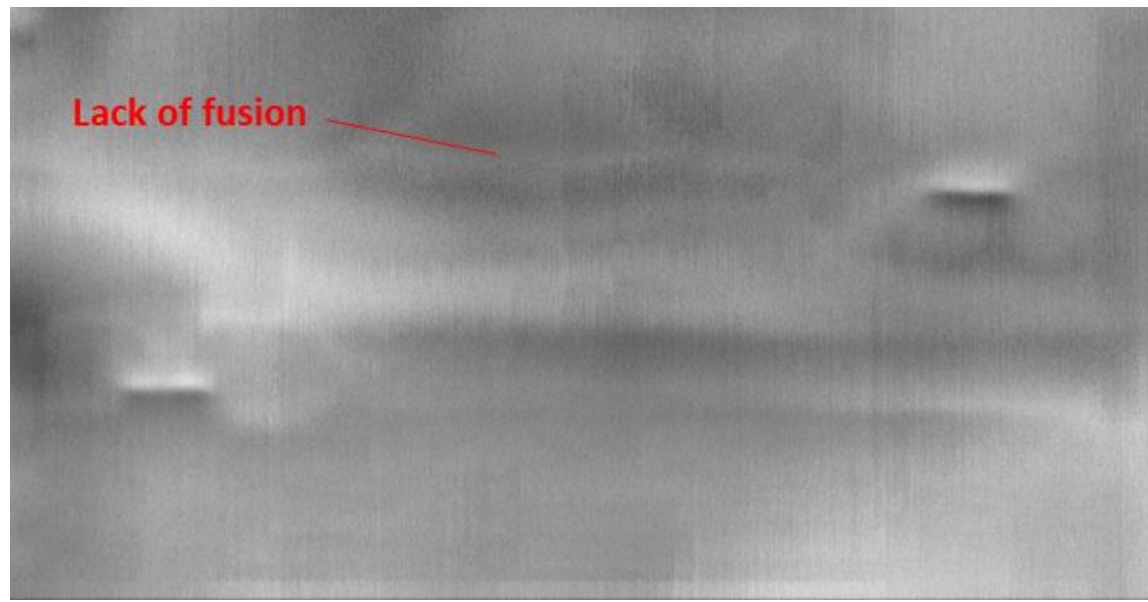

Figure 5. Selection of QWHE magnetic images at different applied magnetic field frequencies and field strengths. Annotated to show weld features.

For each of these 40 magnetic images, the MFL signal to background weld signal ratio $\beta$ for each flaw was calculated, along with the $\bar{\beta}$ value for each image. These are plotted in Figure 6 below: 
(a) Flaw 1

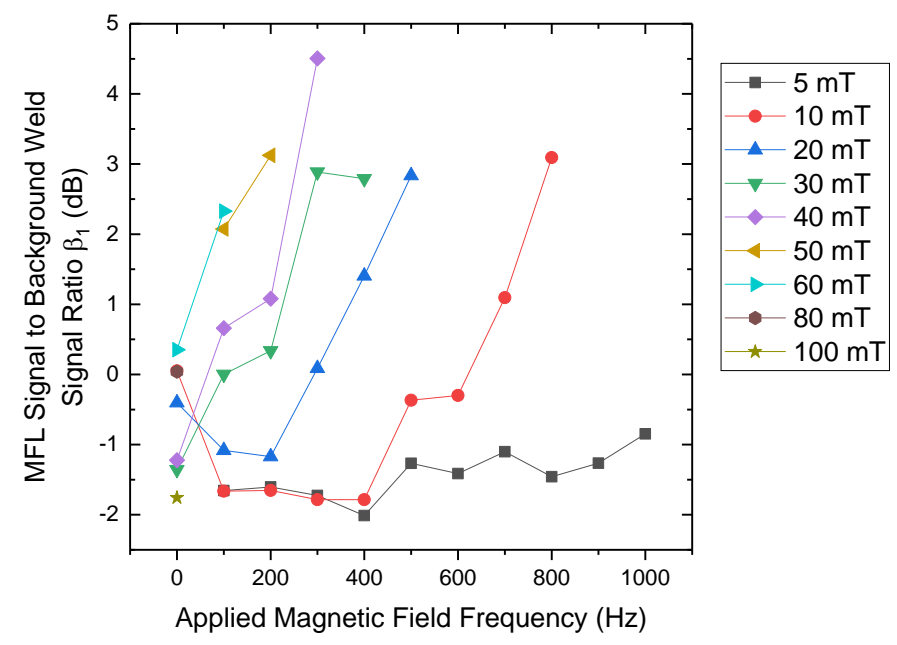

(b) Flaw 2

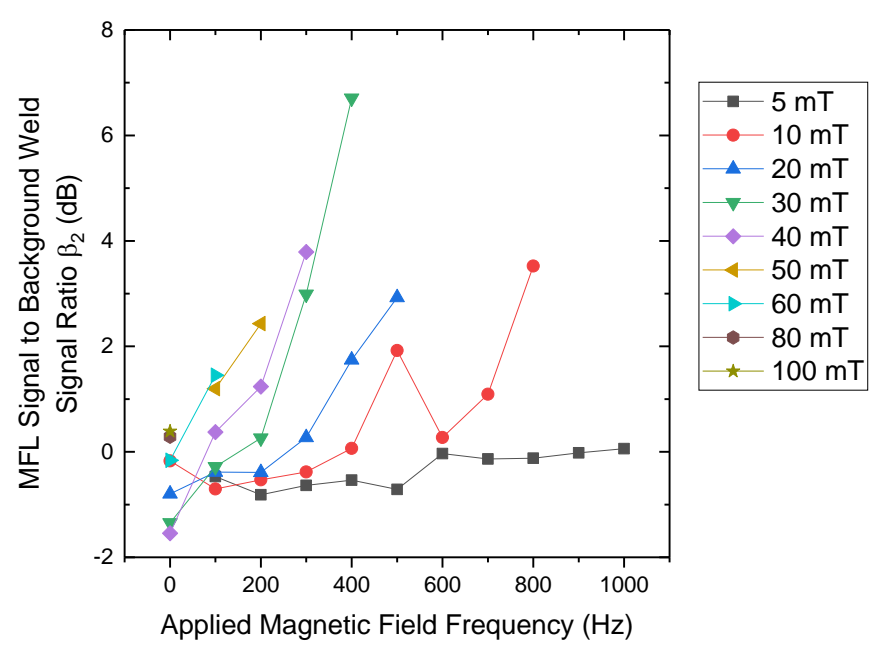

(c) Mean

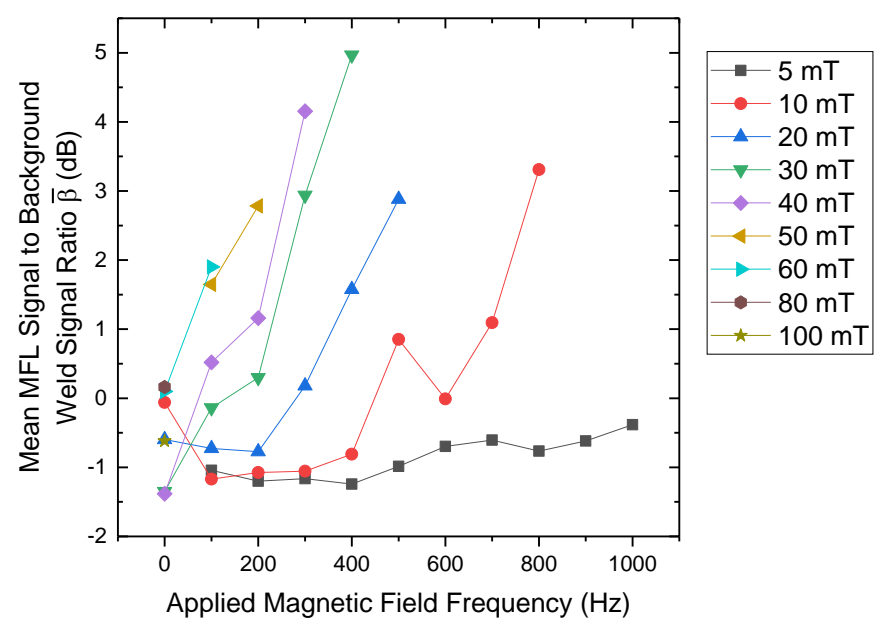

Figure 6. Plots of $\beta_{1}, \beta_{2}$ and $\bar{\beta}$ for the various applied magnetic field frequency and field strength combinations. 
From Figure 6 above it is clear that, in general, the $\beta_{2}$ values were higher than $\beta_{1}$, which can also be seen in the images shown in Figure 5 where Flaw 2 appears whiter than Flaw 1. Also, it must be emphasised that the $\beta$ values do not portray the "true" signal to noise ratio of the QWHE XYZ scanner or the MFL technique in general, since they could be dramatically increased by reducing the region of interest of Flaw 1 and Flaw 2, giving higher $\alpha$ and therefore higher $\beta$ values too. As such, $\beta$ values are used only to quantitatively compare the 40 images within this study data set.

Figure $6 \mathrm{c}$ also shows two particular applied magnetic field frequency and field strength regions of interest: 300 to $400 \mathrm{~Hz}$ at 40 and $30 \mathrm{mT}$ respectively, as well as $800 \mathrm{~Hz}$ at 10 mT. Based on these, the power dissipations, $W_{\text {coil }}$, for each applied magnetic field frequency and field strength combination were calculated using initial control calibrations of the XYZ scanning system. This was deemed important in establishing if an optimum frequency-field range exists, since although the $\bar{\beta}$ values for $300 \mathrm{~Hz} 40 \mathrm{mT}$ and $400 \mathrm{~Hz}$ $30 \mathrm{mT}$ were higher than those of $800 \mathrm{~Hz} 10 \mathrm{mT}$, they required a much higher power to achieve this.

The power dissipation in the coil $\left(W_{\text {coil }}\right)$ values were then plotted along with the other inspection parameters including applied magnetic field strength, $\bar{\beta}$ and applied magnetic field frequency as shown in Figure 7 below:

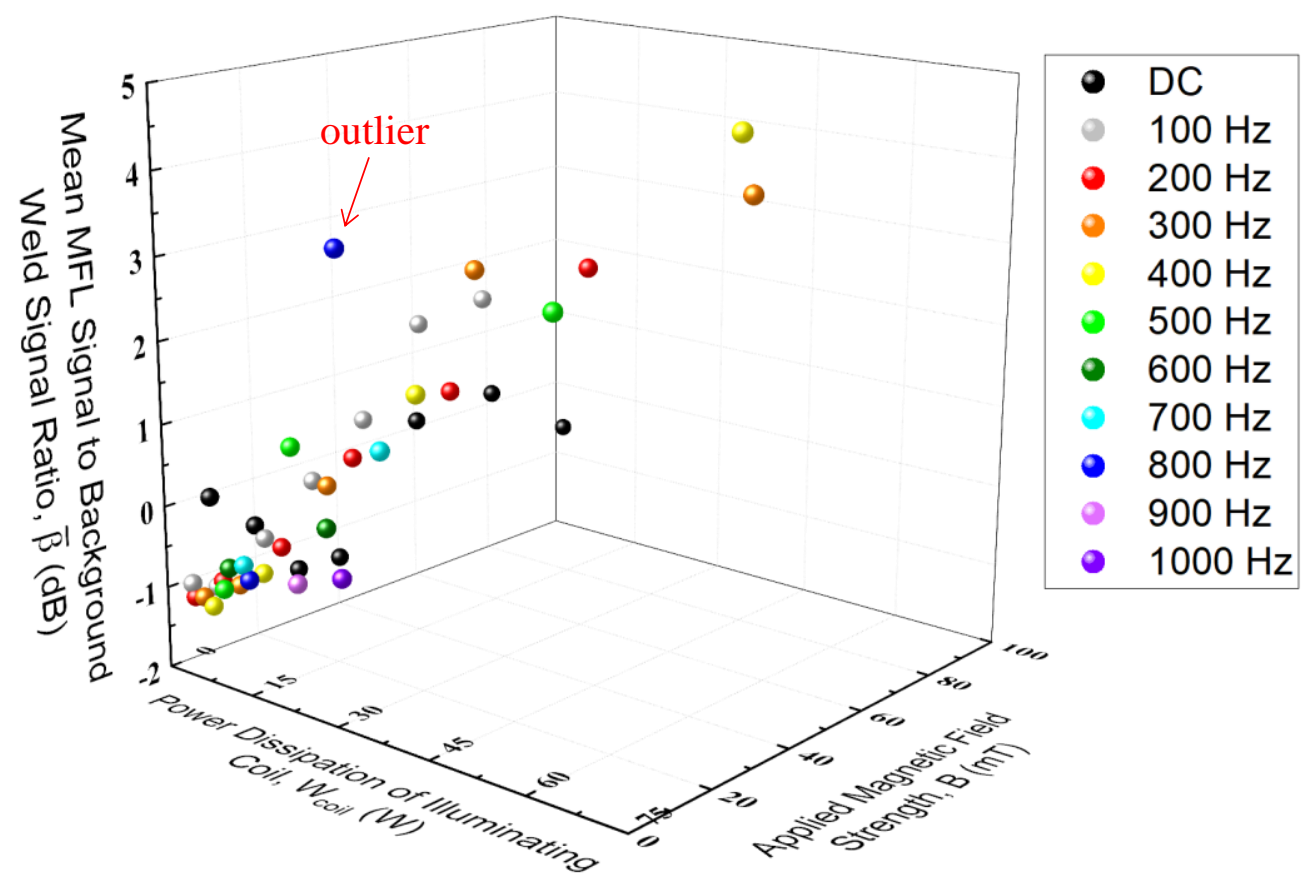

\section{Figure 7. Plot of $\bar{\beta}$ values against applied magnetic field frequency and field strength, as well as power dissipation of the coil.}

Figure 7 above clearly shows a general trend where the low power, low strength applied magnetic fields have a substantially low $\bar{\beta}$. This also includes the higher frequency 
combinations where only low strength fields can be applied due to the larger coil impedance.

More importantly, Figure 7 suggests that the outlier of applying $800 \mathrm{~Hz}$ at $10 \mathrm{mT}$ is optimal, based on the comparatively higher $\beta$ from both flaws, along with considerably lower $(<35 \%)$ power consumption requirements of applied magnetic field frequency and field strength combinations with comparable or higher $\bar{\beta}$ values. Although it is out of the scope of this work to prove any explanation of the study findings, some conjectures are discussed next.

Using electrical conductivity and magnetic permeability values of AISI 1010, the skin depths at $800 \mathrm{~Hz}, 900 \mathrm{~Hz}$ and $1000 \mathrm{~Hz}$ are approximately $210 \mu \mathrm{m} 200 \mu \mathrm{m}$ and $190 \mu \mathrm{m}$ respectively. The differences between these are negligible. Therefore, it can be suggested that the $800 \mathrm{~Hz} 10 \mathrm{mT}$ has effectively double the magnetic field strength confined (since the field strengths were $5 \mathrm{mT}$ for both the $900 \mathrm{~Hz}$ and $1000 \mathrm{~Hz}$ frequencies), producing more leakage near the flaw-sample boundaries. Therefore, it can be argued that the illumining coil parameters of the QWHE XYZ scanner are potentially biased/optimised to $800 \mathrm{~Hz}$ : in this instance being the only low power, high frequency, higher-strength (> $5 \mathrm{mT}$ ) applied field combination possible with the illuminating coil parameters. In which case, the optimisation is not an underlying MFL principle; and that different illuminating coil parameters may respond with a different optimal frequency-field range. However, the $\bar{\beta}$ values for $600 \mathrm{~Hz}$ and $700 \mathrm{~Hz}$ at $10 \mathrm{mT}$ are significantly lower than $800 \mathrm{~Hz}$, despite having approximate skin depths of $245 \mu \mathrm{m}$ and $225 \mu \mathrm{m}$ respectively, being not too dissimilar to $800 \mathrm{~Hz}$.

It can be argued that since this $800 \mathrm{~Hz} 10 \mathrm{mT}$ combination is the only low power, high frequency, higher-strength applied field combination, under these conditions the flaws will be subjected to more eddy currents and hence a better detectability. However, it must be emphasised that the footprint of the applied magnetic field is extremely localised: 5 $\mathrm{mm} \times 15 \mathrm{~mm}$, and is orientated perpendicular to the major axis of the flaws. Consequently, the eddy currents produced will not be disrupted by the flaws as they will be orientated parallel to the flaws, flowing in the z-y plane shown in Figure 2. The eddy current secondary magnetic fields, although orientated perpendicular to the flaw, will also not produce an MFL response since they will be in the opposing direction of the applied magnetic field.

Alternatively, it can be also speculated that at $800 \mathrm{~Hz}$ the magnetic permeability differences between the parent steel plates, the weld metal and microstructures in the heat affected zone are dramatically reduced. This in turn leads to much lower MFL responses from the weld and other contributors of the background weld signal, giving higher $\bar{\beta}$ values. This proposal also explains why the $600 \mathrm{~Hz}$ and $700 \mathrm{~Hz} 10 \mathrm{mT}$ measurements had significantly lower $\bar{\beta}$ values despite also having similar skin depths (not considered too different to warrant the 3 to $2 \mathrm{~dB}$ differences respectively). Figure 6 also suggests $600 \mathrm{~Hz}$ as an inflexion point, where the $\bar{\beta}$ values for the same strength applied magnetic field start to increase dramatically with frequency after this point. 
It must be noted that although these measurements and analysis suggest an optimum frequency and field strength range, this is only applicable to the measurement set up used, along with numerous other inspection parameters including:

- Flaw type.

- Flaw size and depth.

- Sample material electrical and magnetic properties.

- Surface finish of sample.

- Illuminating coil parameters (number of turns, core material, driver current, etc.).

- Applied magnetic field footprint.

- Lift-off distance.

Repeating this study and adding new data points to Figure 6, as well as being able to determine the variance between $\bar{\beta}$ values of the same applied magnetic field frequency and field strength values, would add more confidence to the investigations carried out here. In addition, it would be of interest to repeat the study using a different length toe crack and different illuminating coil parameters to observe any effect that these would have on an optimum applied magnetic field frequency and field strength range. Furthermore, it would also be of interest to repeat the study using different electromagnet parameters to better suit $>5 \mathrm{mT}$ fields at $>1 \mathrm{kHz}$, namely decreasing the number of turns and using a core material with less hysteresis loss (such as composite or ferrite) to test some of the conjectures put forward to explain the results of the study. 


\title{
4. Conclusions
}

In this work, 40 magnetic images using a QWHE sensor XYZ scanner were obtained with various applied magnetic field frequency and field strengths across the complete operational range of the scanner.

Quantitative analysis was then performed to determine that an applied magnetic field frequency of $800 \mathrm{~Hz}$ of $10 \mathrm{mT}$ rms strength was optimal for the detection of the $11 \mathrm{~mm}$ and $10 \mathrm{~mm}$ length surface-breaking toe cracks in a ground mild steel weld sample. This applied magnetic field frequency and field strength pair was the result of the combination of comparatively high detectability and low power consumption of the illuminating coil.

Future work being undertaken to develop the maturity of the QWHE imaging technique includes:

- Repeating the study, using smaller length toe cracks in the same sample material to ascertain the effect of flaw size on the optimum frequency.

- Repeating the study, using a much larger applied magnetic field footprint to ascertain the effect of footprint on the optimum frequency.

- Repeating the study, using various lift-off values to measure the effect of lift-off for MFL imaging using QWHE sensors.

- Processing the dataset from this study to determine a minimum / optimum measurement step where the flaws are still detectable (for optimum sensor array pitch).

- Developing enhanced, optimised imaging technology based on this underpinning research.

- Developing image enhancement techniques and automated flaw detection and sizing algorithms using frequency-based analysis and/or spatial MFL field distribution around flaws.

- Building a database of MFL responses from flaws of known dimensions for future enhanced characterisation and reconstruction.

- Developing multi-frequency / pulsed applied magnetic field technologies for detection, imaging, sizing and characterisation of surface-breaking and nearsurface flaws ( $<3 \mathrm{~mm}$ from surface).

\section{Acknowledgements}

\author{
EPSRC-EP/P006973/1 "FUTURE COMPOUND SEMI-CONDUCTOR \\ MANUFACTURING HUB”) \\ EPSRC-EP/LO22125/1 "UK RESEARCH CENTRE IN NON-DESTRUCTIVE \\ EVALUATION(RCNDE3)"
}

ICASE PhD STUDENTSHIP THROUGH RCNDE (BAE Systems) 


\section{References}

1. British Standard BS EN ISO 9934-1:2016. Non-destructive testing - Magnetic particle testing. Part 1: General principles (ISO 9934-1:2016), 2016.

2. British Standard BS EN ISO 15549:2010. Non-destructive testing - Eddy current testing - General principles, 2010.

3. S. S. Udpa, 'NDT Handbook - Electromagnetic Testing' (3rd ed.). Columbus: American Society for Nondestructive Testing, 2004.

4. N. Haned and M. Missous, 'Nano-tesla magnetic field magnetometry using an InGaAs-AlGaAs-GaAs 2DEG Hall sensor', Sensors and Actuators, 102(3), 216222. DOI 10.1016/S0924-4247(02)00386-2, 2003.

5. J. M. Watson, C.W. Liang, E. Ahmad, J. Sexton and M. Missous, 'Surface Crack Detection in Dressed Steel Welds Using Advanced Quantum Well Hall Effect Sensors', 56th Annual Conference of the British Institute of Non-Destructive Testing, 2017.

6. J. M. Watson, C.W. Liang, J. Sexton, F. A. Biruu and M. Missous, 'A Comparative Study of Electromagnetic NDE Methods and Quantum Well Hall Effect Sensor Imaging for Surface-Flaw Detection in Mild Steel Welds', 57th Annual British Conference on Non-Destructive Testing, 2018.

7. J. M. Watson, C.W. Liang, J. Sexton, F. A. Biruu and M. Missous, 'SurfaceBreaking Flaw Detection in Ferritic Welds using Quantum Well Hall Effect Sensor Devices', 45th Annual Review of Progress in Quantitative Nondestructive Evaluation, 2018.

8. C. W. Liang, E. Ahmad, E. Balaban, F. A. Biruu, J. Sexton and M. Missous, 'A Real Time Quantum Well Hall Effect 2D Handheld Magnetovision System for Ferromagnetic and Non-Ferromagnetic Materials Nondestructive Testing', 56th Annual Conference of the British Institute of Non-Destructive Testing, 2017.

9. E. Ahmad, J. Watson, C. W. Liang, J. Sexton and M. Missous, 'RCNDE Core Research Review Report: July 2018. Sub-Project 1.3: Magnetic Camera', RCNDE Board, 2018. 